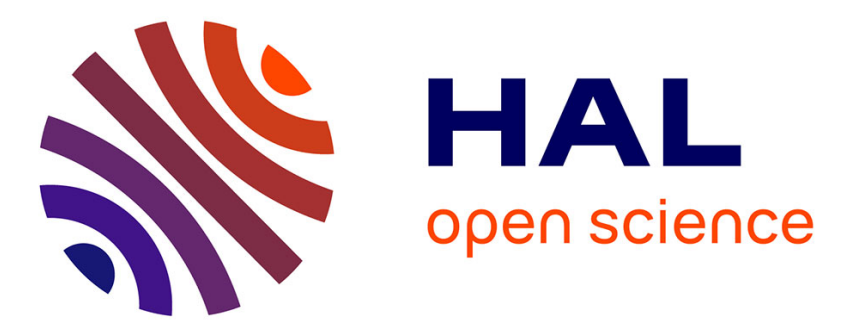

\title{
Modulation instability in the weak normal dispersion region of passive fiber ring cavities
}

F. Bessin, F. Copie, Matteo Conforti, Alexandre Kudlinski, Arnaud Mussot

\section{To cite this version:}

F. Bessin, F. Copie, Matteo Conforti, Alexandre Kudlinski, Arnaud Mussot. Modulation instability in the weak normal dispersion region of passive fiber ring cavities. Optics Letters, 2017, 42 (19), pp.3730-3733. 10.1364/OL.42.003730 . hal-02386056

\section{HAL Id: hal-02386056 https://hal.science/hal-02386056}

Submitted on 29 Nov 2019

HAL is a multi-disciplinary open access archive for the deposit and dissemination of scientific research documents, whether they are published or not. The documents may come from teaching and research institutions in France or abroad, or from public or private research centers.
L'archive ouverte pluridisciplinaire HAL, est destinée au dépôt et à la diffusion de documents scientifiques de niveau recherche, publiés ou non, émanant des établissements d'enseignement et de recherche français ou étrangers, des laboratoires publics ou privés. 


\title{
Modulation instability in the weak normal dispersion region of passive fiber ring cavities
}

\author{
F. Bessin ${ }^{1,}$, F. Copie ${ }^{1}$, M. Conforti ${ }^{1}$, A. Kudlinski ${ }^{1}$, And A. Mussot ${ }^{1}$ \\ ${ }^{1}$ Université Lille, CNRS, UMR 8523-PhLAM-Physique des Lasers Atomes et Molécules, F-59000 Lille, France \\ ${ }^{*}$ Corresponding author: florent.bessin@ed.univ-lille1.fr
}

We report the experimental observation of modulation instability in the weak normal dispersion region of a passive fiber ring cavity. We show that the fourth-order dispersion strongly modifies the dynamics of the cavity through the generation of new instability bands. Experimental results are in excellent agreement with theoretical predictions and numerical simulations. () 2017 Optical Society of America

OCIS codes: (190.4370) Nonlinear optics, fibers; (190.3100) Instabilities and chaos; (230.5750) Resonators

http://dx.doi.org/10.1364/ao.XX.XXXXXX

Modulation instabilty (MI) in passive optical cavities has been widely studied during the last 30 years and has found a wealth of applications ranging from ultra-fast pulse train generation to frequency combs [1]. MI results from the interplay between dispersion, nonlinearity and cavity detuning and all these parameters appear in the phase matching relation that characterizes the process. To date, most investigations have been carried out in large dispersion regions where the Taylor development of the propagation constant limited to the second-order (the group velocity dispersion, $\beta_{2}$ ) is enough to capture the whole dynamics of the system [2, 3]. In weak dispersion regions, since the width of MI spectra is inversely proportional to the group velocity dispersion [4], the spectrum is wider and it is thus necessary to consider higher order dispersion terms to account for the variation of the propagation constant over the whole spectrum. Consequently, it has been shown that even orders have to be considered in the phase matching relation [5], as in the single pass configurations [6-8]. These additional terms allow new phase matching frequencies, which strongly modify the dynamics of the system. For instance it allows MI to exist in the normal dispersion region of single pass configurations $[7,8]$, or more surprisingly in passive cavities, leads to the disappearance of MI when the pump power exceeds a certain value [5] or to the generation of tens of quasi-phase-matched MI side lobes in dispersion varying cavities [9]. Note that odd orders dispersion terms do not enter into play in the phase matching relation that characterizes the linear stage of MI (just above the MI threshold), but they have an impact on the nonlinear stage of MI. For instance, they lead to a symmetry breaking of the MI spectrum $[10,11]$, or to the generation of dispersive waves in the context of cavity solitons [12], which stabilize the underlying Kerr frequency combs [13].

In this letter we present an experimental study of MI in the weak normal dispersion region of a passive fiber ring cavity in both the monostable and bistable regimes in the linear stage of the process (just above the MI threshold). We show that taking into account the fourth-order dispersion is required to capture the whole dynamics of cavities operating in this regime. It leads to significant modifications of the MI process among which, the generation of new spectral sidebands. Experimental results are in excellent agreement with theoretical predictions and numerical simulations.

Light propagation in passive fiber ring cavities is well modelled by the Lugiato-Lefever equation (LLE) [4, 14]. In low dispersion regions, it is necessary to account for higher order dispersion terms leading to an extended version of the original LLE $[5,9,15]$ :

$$
i \frac{\partial E}{\partial z}+\sum_{n=2}^{4} i^{n} \frac{\beta_{n}}{n !} \frac{\partial^{n} E}{\partial t^{n}}+\gamma|E|^{2} E=\left(\frac{\delta}{L}-i \frac{\alpha}{L}\right) E+i \frac{\theta}{L} E_{i n}
$$

where $z$ is the propagation distance, $t$ the time in the frame travelling at the group velocity dispersion of the pump, and $E$ the intracavity electric field envelop. $L$ is the cavity length, $\gamma$ the nonlinear coefficient of the fiber. $\beta_{2}, \beta_{3}$, and $\beta_{4}$ are respectively the group velocity dispersion, the third-order and the fourth-order dispersion terms. Note that we restricted the development up to $\beta_{4}$ because higher order contribution is negligible regarding the MI frequency shifts observed in realistic passive cavities. $E_{i n}$ is the input field driving the cavity. $\theta$ and $\rho$ define, respectively, the coupler transmission and reflection coefficients such that $\theta^{2}+\rho^{2}=1$. $\delta$ refers to the cavity detuning which can be normalized in the following way $\Delta=\frac{\delta}{\alpha}$ [4]. In this way, the system is known to be monostable whenever $\Delta<\sqrt{3}$, and bistable otherwise $[4,14]$. The finesse of the cavity is usually defined as $F=\frac{\pi}{\alpha}$ where $\alpha$ describes the total losses (splices, linear, coupling and excess losses of the coupler) over one round-trip of the cavity. The parametric gain can be calculated from a linear stability analysis, as described in Ref. [5] which gives the most unstable frequencies:

$$
\omega=\sqrt{\frac{-6 \beta_{2} \pm 6 \sqrt{\beta_{2}^{2}-\frac{2}{3} \beta_{4}\left(2 \gamma P-\frac{\delta}{L}\right)}}{\beta_{4}}}
$$



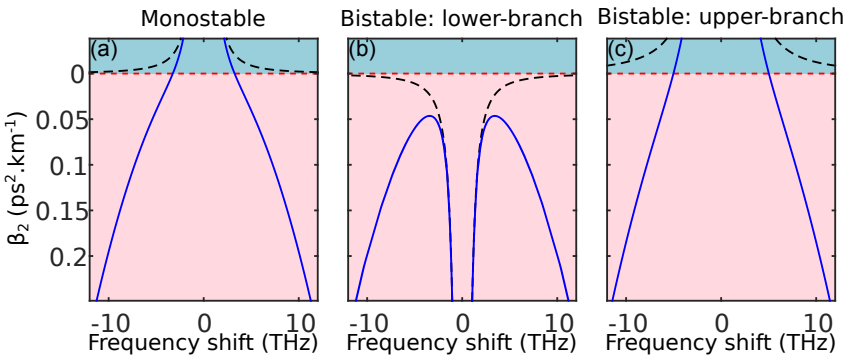

Fig. 1. Frequency shifts calculated from Eq.(2) with $\beta_{4}$ (solid blue lines) and without (dashed black lines). (a) In the monostable regime $\left(\Delta=1.1, P_{\text {in }}=230 \mathrm{~mW}\right)$, in the bistable regime $(\Delta=4.6)(\mathrm{b})$ on the lower branch $\left(P_{i n}=2.69 \mathrm{~W}\right)$ and (c) on the upper branch $\left(P_{\text {in }}=4.09 \mathrm{~W}\right)$. Red (blue) area: normal (anomalous) dispersion region. Parameters as in experiments: $\gamma=2.5$ $\mathrm{W}^{-1} \cdot \mathrm{km}^{-1}, \beta_{4}=-6.0 \times 10^{-4} \mathrm{ps}^{4} \cdot \mathrm{km}^{-1}, L=37.9 \mathrm{~m}$.

In the following, we focus our attention on configurations for which $\beta_{4}<0$ as it is the case in most optical fibers and when $\beta_{2}>0$. We do not investigate the case where $\beta_{2}$ is also negative since only the position of MI sidebands would be affected without impacting the MI dynamics contrary to what will be shown in this paper. Note that the specific case where $\beta_{4}>0$ and $\beta_{2}<0$ has been addressed theoretically in Ref. [5], and show in particular that two frequencies can be destabilized at a primary intra-cavity power threshold. When the input power increases, the two corresponding bands of unstable frequencies widen, and merge in a single large band to eventually disappear from a secondary pump threshold, the system returning to a stationary state. Figure 1 illustrates the evolution of the most unstable frequencies calculated from Eq.(2) for both regimes of operation of the cavity (Fig. 1 (a) monostable and Fig. 1 bistable, lower (b) and upper (c) branch respectively). We represent this evolution across normal $\left(\beta_{2}>0\right)$ and anomalous $\left(\beta_{2}<0\right)$ dispersion regions with $\beta_{4}$ (solid blue lines) and without $\beta_{4}$ (dashed black lines). The normalized cavity detuning has been fixed to $\Delta=1.1$ for the monostable regime and $\Delta=4.6$ for the bistable regime, which is above the critical value of 4.25 that allows MI to be stably observed on the lower branch [16]. The impact of $\beta_{4}$ can be divided into two main categories. In the first one, without $\beta_{4}$ MI only exits in the anomalous dispersion region (dashed black lines) while with $\beta_{4}$, owing to its negative value, a perfect phase matching exists whatever the sign of $\beta_{2}$. This occurs in the monostable regime (Fig. 1 (a)) or on the upper branch of the bistable regime (Fig. 1 (c)). The second category corresponds to the lower branch of the bistable regime (Fig. 1 (b)). In this regime, $\beta_{4}$ leads to the generation of a second pair of MI side lobes (solid blue lines) while without $\beta_{4}$ a single pair is predicted (dashed black lines). Consequently, the contribution of $\beta_{4}$ on the dynamics of passive fiber cavities is very important as it allows the observation of MI in new windows of dispersion (normal dispersion in monostable regime and in the upper branch of the bistable one) and leads to the generation of new MI sidebands in the lower branch of the bistable regime.

In order to validate these theoretical predictions experimentally, we built a cavity for which the group velocity dispersion is slightly normal at the pump wavelength. The experimental setup is depicted in Fig. 2 and is similar to the one reported in Ref. $[9,17]$ except that the cavity is uniform and made of a dispersion shifted fiber (DSF). Investigation of MI in this cavity is performed by means of a pulse train whose wavelength is tunable between $1549.5 \mathrm{~nm}$ and $1550.5 \mathrm{~nm}$. These pulses are generated from a continuous wave $(\mathrm{cw})$ laser which is chopped by an electro-optic modulator (EOM) with a repetition rate of $5.4 \mathrm{MHz}$ corresponding to the round trip time of the $37.9 \mathrm{~m}$ long cavity. Brillouin scattering has been avoided by using square pulses with a duration of $1.5 \mathrm{~ns}$. These pulses are amplified by means of an erbium doped fiber amplifier (EDFA), and then filtered to remove amplified spontaneous emission (ASE) in excess. The cavity is built with a DSF and a 90/10 coupler made of the same fibers to get a perfectly uniform cavity and to avoid instabilities that might be observed in dispersion varying cavities $[9,17,18]$. The DSF has a zero dispersion wavelength (ZDW) at $1551.6 \mathrm{~nm}$ and a nonlinear coefficient $\gamma$ of $2.5 \mathrm{~W}^{-1} \cdot \mathrm{km}^{-1}$. The pump wavelength has been tuned between $1549.5 \mathrm{~nm}$ and 1550.5 $\mathrm{nm}$ corresponding to a weak positive group velocity dispersion variation lying between 0.20 and $0.11 \mathrm{ps}^{2} \cdot \mathrm{km}^{-1}$. Higher order dispersion terms are $\beta_{3}=0.12 \mathrm{ps}^{3} \cdot \mathrm{km}^{-1}$ and $\beta_{4}=-6.0 \times 10^{-4}$ $\mathrm{ps}^{4} \cdot \mathrm{km}^{-1}$. Two circulators at each output of the cavity allows us to use independently the two propagation directions of the cavity. Pump pulses propagate in the anti-clockwise direction (blue arrows Fig. 2), while a fraction of the cw laser travels in the clockwise direction (green arrows Fig. 2). This latter is used to stabilize the cavity length against external perturbations by using a feedback loop (proportional-integral-derivative controller, PID). As in Ref. $[3,9,17,19]$ a combination of three polarization controllers are used to control the cavity detuning.

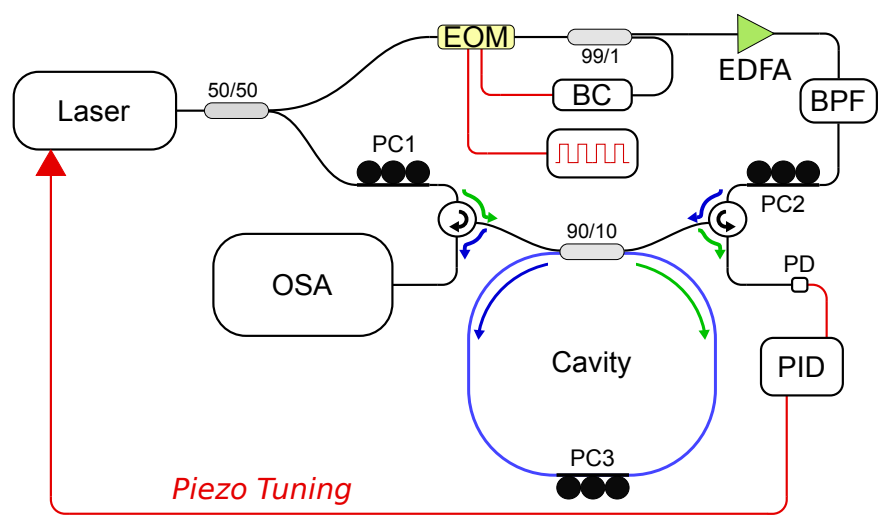

Fig. 2. Experimental setup. $P C$, polarization controller; $B C$, bias controller; PD, photo-detector; EOM, electro-optic modulator; OSA, optical spectrum analyser; EDFA, erbium-doped fiber amplifier; BPF, band-pass filter; PID, proportionalintegral-derivate controller.

We first analyse the monostable regime by setting the detuning to $\delta=0.13 \mathrm{rad}$. The cavity finesse is estimated to be $F=26.5$ which corresponds to total losses $\alpha=0.118$. Accordingly, the normalized detuning is $\Delta=\delta / \alpha=1.1<\sqrt{3}[16,17]$. We set the pump wavelength at $1549.9 \mathrm{~nm}(1.7 \mathrm{~nm}$ below the ZDW leading to $\left.\beta_{2}=0.16 \mathrm{ps}^{2} \cdot \mathrm{km}^{-1}\right)$ just above the MI threshold of the cavity with $P_{\text {in }}=0.23 \mathrm{~W}\left(P_{\text {in }, \text { threshold }}=\alpha^{3} /\left(\gamma \theta^{2} L\right)\left(\Delta^{2}-2 \Delta+2\right)=\right.$ $0.18 \mathrm{~W}$ from theoretical predictions). As can be seen in Fig. 3(a), two symmetric side lobes are generated on both sides of the pump at 9.28 THz. These experimental results are in excellent agreement with theoretical predictions from Eq. (2), (9.25 THz, indicated by a vertical dashed line) and from numerical simulations of the LLE (Eq. (1), Fig. 3(b)). In order to validate the theoretical predictions of Eq. (2) regarding the evolution of the position of the MI sidebands with the group velocity dispersion, 


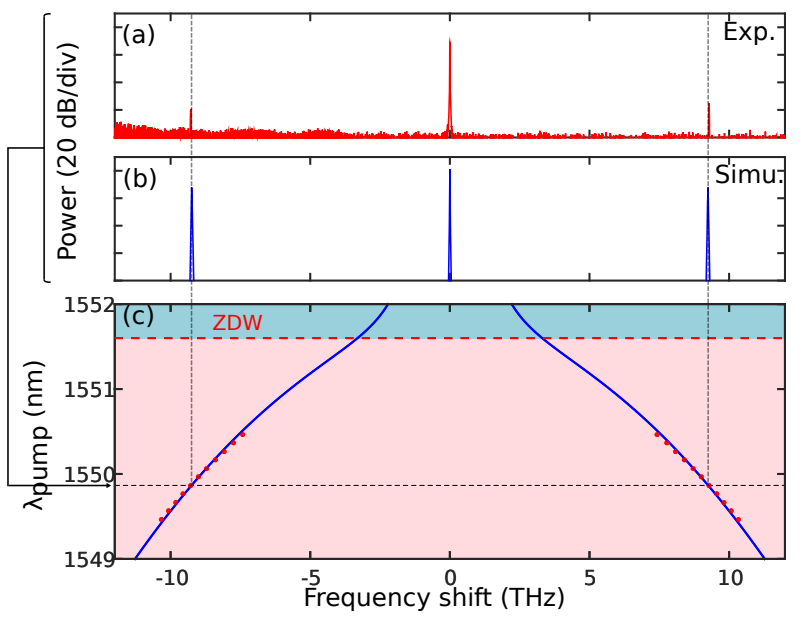

Fig. 3. Cavity output spectra in the monostable regime. (a) experiments, (b) numerics from Eq. 1. (c) Evolution of MI sidebands position as a function of the pump wavelength, red dots from experimental measurements and solid blue lines from theory (Eq. 2). Parameters: $\Delta=1.1<\sqrt{3}, \lambda_{\text {pump }}=$ $1549.9 \mathrm{~nm}$ and $P_{\text {in }}=0.23 \mathrm{~W}$.

we recorded output spectra for pump wavelengths ranging from $1549.5 \mathrm{~nm}$ to $1550.5 \mathrm{~nm}$. Experimental results are represented by red dots in Fig. 3(c) and theoretical predictions (Eq. (2)) by solid blue lines. By moving closer to the ZDW of the fiber, the frequency shift of the MI sidebands is reduced, in excellent agreement with theoretical predictions.

Then, we investigated the bistable regime of the cavity. The pump power has been set to $2.69 \mathrm{~W}$, just above the MI threshold of the lower branch of the bistable cycle. As expected from the theory, two pairs of MI side lobes have been generated on both sides of the pump (Fig 4(a)) by pumping in the weak normal dispersion region of the fiber $\left(\lambda_{\text {pump }}=1549.9 \mathrm{~nm}, 1.7 \mathrm{~nm}\right.$ below the ZDW). They are located at $1.18 \mathrm{THz}$ and $9.19 \mathrm{THz}$ respectively. Note that we also see the birth of harmonics of the first sideband pair at $2.36 \mathrm{THz}$ due to degenerate four-wave mixing (FWM) with the pump. Again, these results are confirmed by theoretical prediction from Eq. $2(1.29 \mathrm{THz}$ and $9.09 \mathrm{THz}$, dashed vertical lines in Figs. 4), and numerical simulations from the LLE (Fig. 4 (b)). It is quite remarkable to note that while the system exibits more than one single pair of sidebands their amplitude seems very similar in both experiments and numerics (Fig. 4(a) and (b)). In order to confirm this specific feature, we calculated the parametric gain spectrum from the linear stability analysis of Eq. (1), which is represented Fig. 4 (c). It reveals that the parametric gain value is indeed exactly the same for each sideband, as found in the case where $\beta_{4}$ is positive [5]. The evolution of the frequency shifts of these sidebands as a function of the pump wavelength has been investigated experimentally (red dots in Fig. 4(c)). The frequency shift of the first sideband pair increases and the one of the second sideband pair decreases as the pump wavelength gets closer to the ZDW, in excellent agreement with theoretical predictions (blue curves). Above $1551.2 \mathrm{~nm}$, no MI is expected to be observed on the lower branch of the bistable cycle (Fig. 4 (c)). This feature is due to the contribution of $\beta_{4}$ since without $\beta_{4}$, MI is predicted in the whole normal dispersion region of the cavity (Fig. 1 (b)).

Finally, MI on the upper branch of the bistable regime of the cavity has been studied. To switch the system over the up-

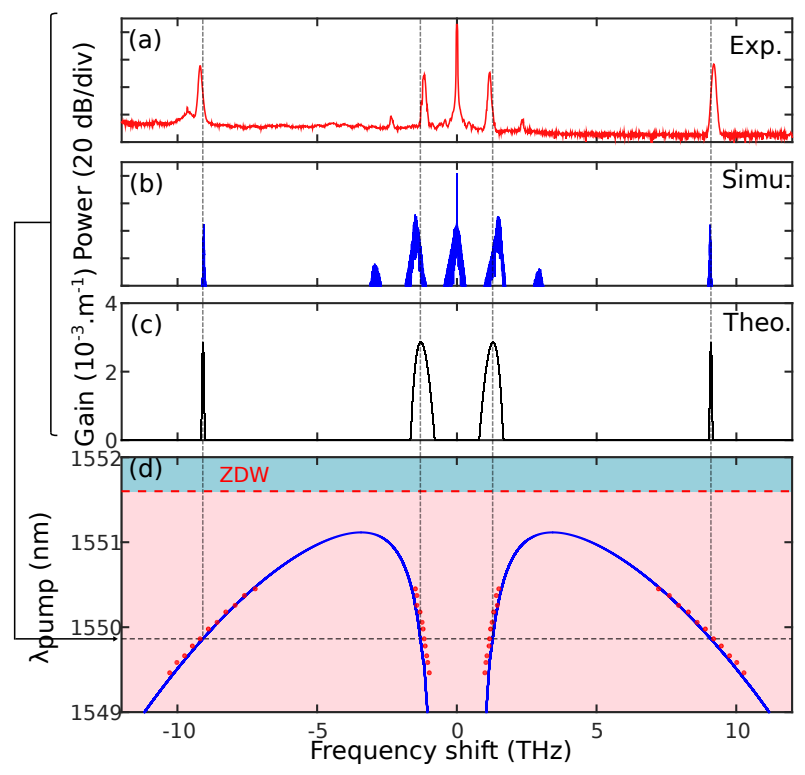

Fig. 4. Cavity output spectra on the lower branch of the bistable regime. (a) experiments, (b) numerics from Eq. 1, (c) theoretical gain spectrum. (d) Evolution of MI sidebands position as a function of the pump wavelength, red dots from experimental measurements and solid blue lines from theory (Eq. 2). Parameters: $\Delta=4.6, \lambda_{\text {pump }}=1549.9 \mathrm{~nm}$ and $P_{\text {in }}=2.69 \mathrm{~W}$.

per branch, the pump power has been increased to $P_{\text {in }}=4.09$ $\mathrm{W}$, which is well above the cavity threshold for this regime $\left(P_{\text {in,threshold }}=2.51 \mathrm{~W}\right)$. Results are reported in Fig. 5, where we used the same representation as in Figs. 3 and Fig. 4. As can be seen, MI indeed exists in the normal dispersion region thanks to the contribution of $\beta_{4}$ (Fig. 5(a)) and the agreement with theory and numerics is good (Fig. 5 (b) and (c)). However, a higher discrepancy is observed in that configuration compared to the previous ones. This might be due to the fact that pump power used is well above the MI threshold in this case. In addition, MI side lobes are asymmetric, this behaviour is due to the third-order dispersion term which induces nonlinear symmetry breaking of the MI process in optical fiber resonators as discussed in [11]. Numerical simulations including this term confirm this asymmetry as it can be seen in Fig. 5(b).

We then address the case of the transitions from the lower to the upper branch of the bistable response of the cavity and vice versa by increasing/decreasing the pump power and recording output steady state spectra. We set the system at $\lambda_{\text {pump }}=1549.7$ $\mathrm{nm}$ with a normalized detuning of $\Delta=5.5$, allowing the observation of MI on both branches [16]. Fig. 6 shows a 2D plot of the evolution of the output spectrum as a function of the pump power. When the pump power is below $P_{\text {in }}=3.75 \mathrm{~W}$, the cavity is stable and no feature of $\mathrm{MI}$ is observed. Above this threshold, and up to $P_{\text {in }}=4.15 \mathrm{~W}$, MI appears on the lower branch and is characterized by two pairs of side lobes. Note that, while being predicted by theory, this steady state regime is very tricky to achieve and only exists for a limited range of parameters (see also Refs [16] and [9, 17]). By further increasing the pump power, the system up-switches on the upper branch of the bistable response of the cavity. The least detuned pair of MI sidebands (at $1.7 \mathrm{THz}$ ) disappears while the other pair broadens and experiences a slight frequency shift. This is in pretty good agreement with what has been discussed and pre- 


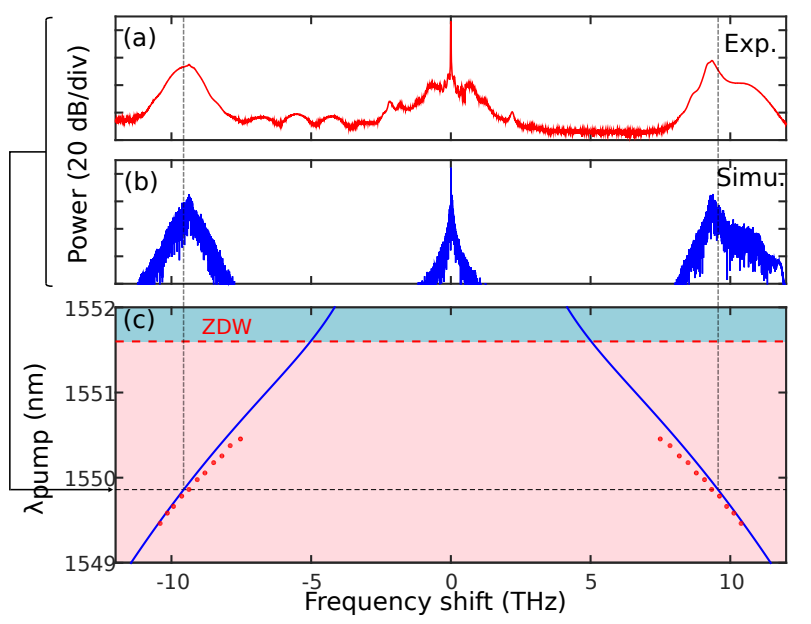

Fig. 5. Cavity output spectra on the upper branch of the bistable regime. (a) experiments, (b) numerics from Eq. 1. (c) Evolution of MI sidebands position as a function of the pump wavelength, red dots from experimental measurements and solid blue lines from theory (Eq. 2). Parameters: $\Delta=4.6$, $\lambda_{\text {pump }}=1549.9 \mathrm{~nm}$ and $P_{\text {in }}=4.09 \mathrm{~W}$.

sented previously. We emphasize than in a cavity with a large normal dispersion, the contribution of $\beta_{4}$ would be negligible and the second pair of sidebands would not exist when working on the lower branch [16, 17]. Also, the cavity would remain stable on the upper branch [16]. Then by decreasing the pump power, the system remains on the upper branch until it down switches at $P_{\text {in }}=2.96 \mathrm{~W}$ (Fig. $6(\mathrm{~d})$ ). This value is lower than the up-switching power which reveals the hysteresis cycle of the cavity. As the MI area on the lower branch is limited to a small range of pump power, the system switches down on the lower branch but on a steady state portion where no MI exists.
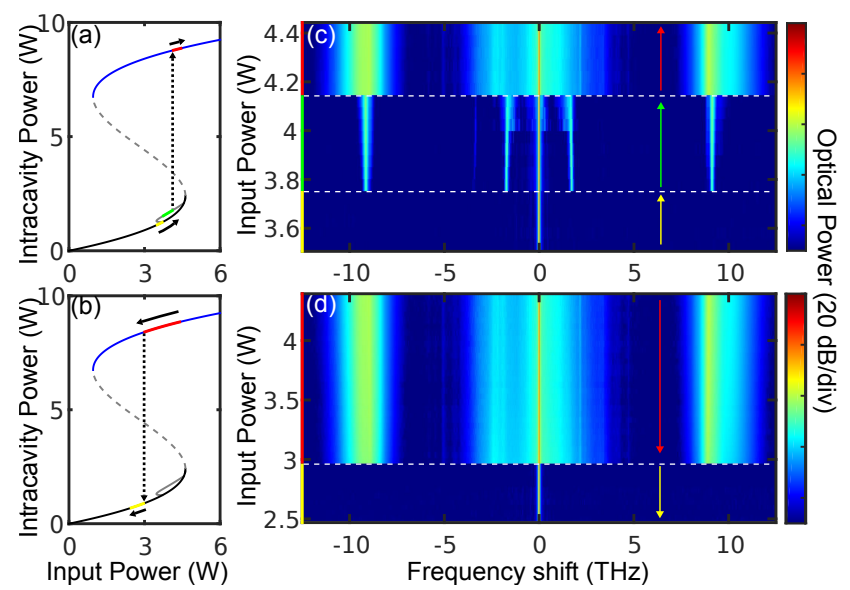

Fig. 6. Color level plot of experimental output cavity spectrum vs input power when increasing input power from the lower branch to the upper branch (c) and to the upper branch to the lower branch (d). (a) and (b), the bistability curve where colored part correspond to the domain investigated on color level plot, colored arrows on (c), (d) correspond respectively to colored part investigated on (a) and (b).

To conclude we report the experimental observation of MI in a passive fiber ring cavity in the weak normal dispersion region.
We show that the fourth-order dispersion term strongly modifies the dynamics of the cavity in both monostable and bistable regimes. In the monostable regime and on the upper branch of the bistable regime, the cavity becomes modulationnaly unstable, and on the lower branch, a second pair of MI sideband is generated owing to the contribution of $\beta_{4}$. Experimental results are confirmed by numerical simulations and are in excellent agreement with theoretical predictions from the LLE. These investigations have been performed in the context of modulation instability, just above the threshold but should also impact nonlinear effects of higher order such as frequency comb/cavity soliton generation, which are involved in a wealth of promising applications [1, 20-22].

\section{ACKNOWLEDGEMENT}

This work was partly supported by IRCICA (USR 3380 Univ. Lille-CNRS), by the Agence Nationale de la Recherche (grants TOPWAVE, NoAWE, HEAFISY, Labex CEMPI and Equipex FLUX), by the French Ministry of Higher Education and Research, the Nord- Pas de Calais Regional Council and Fonds Européen de Développement Économique Régional (grant CPER Photonics for Society).

\section{REFERENCES}

1. P. Grelu, Nonlinear Optical Cavity Dynamics: From Microresonators to Fiber Lasers, (Wiley-VCH 2015).

2. M. Nakazawa, K. Suzuki, and H. A. Haus, Phys. Rev. A. 38, 5193-5196 (1988).

3. S. Coen, and M. Haelterman, Phys. Rev. Lett. 79, 4139-4142 (1997)

4. M. Haelterman, S. Trillo, and S. Wabnitz, Opt. Lett. 17, 745-747 (1992).

5. M. Tlidi, A. Mussot, E. Louvergneaux, G. Kozyreff, A. G. Vladimirov, and M. Taki, Opt. Lett. 32, 662 (2007).

6. S. B. Cavalcanti, J. C. Cressoni, H. R. da Cruz, and A. S. Gouveia-Neto, Phys. Rev. A 43, 6162-6165 (1991).

7. S. Pitois and G. Millot, Opt. Commun. 226, 415-422 (2003).

8. J. D. Harvey, R. Leonhardt, S. Coen, and G. K. L. Wong, Opt. Lett. 28 , 2225-2227 (2003).

9. F. Copie, M. Conforti, A. Kudlinski, S. Trillo, and A. Mussot, Opt. Express 25, 11283-11296 (2017).

10. A. Mussot, E. Louvergneaux, N. Akhmediev, F. Reynaud, L. Delage, and M. Taki, Phys. Rev. Lett. 101, 113904 (2008).

11. F. Leo, A. Mussot, P. Kockaert, P. Emplit, M. Haelterman, and M. Taki, Phys. Rev. Lett. 110, 104103 (2013).

12. J. K. Jang, M. Erkintalo, S. G. Murdoch, and S. Coen Opt. Lett. 39, 5503-5506 (2014).

13. P. Parra-Rivas, D. Gomila, F. Leo, S. Coen, and L. Gelens, Opt. Lett. 39, 2971-2974 (2014)

14. L. A. Lugiato and R. Lefever, Phys. Rev. Lett. 58, 2209-2211 (1987).

15. S. Coen, H. G. Randle, T. Sylvestre, and M. Erkintalo, Opt. Lett. 38, 37 (2013).

16. S. Coen, M. Haelterman, P. Emplit, L. Delage, L. M. Simohamed, and François Reynaud, J. Opt. B Quantum Semiclassical Opt. 1, 36 (1999).

17. F. Copie, M. Conforti, A. Kudlinski, and A. Mussot, Phys. Rev. Lett. 116, 143901 (2016).

18. M. Conforti, A. Mussot, A. Kudlinski, and S. Trillo, Opt. Lett. 39, 4200 (2014).

19. S. Coen, M. Haelterman, P. Emplit, L. Delage, L. M. Simohamed, and F. Reynaud, J. Opt. Soc. Am. B 15, 2283 (1998).

20. Y. Okawachi, M. R. E. Lamont, K. Luke, D. O. Carvalho, M. Yu, M. Lipson, and A. L. Gaeta, Opt. Lett. 39, 3535-3538 (2014).

21. C. Milián, A. V. Gorbach, M. Taki, A. V. Yulin, and D. V. Skryabin, Phys. Rev. A 92, 033851 (2015).

22. K. Y. Yang, K. Beha, D. C. Cole, X. Yi, P. Del'Haye, H. Lee, J. Li, D. Y. Oh, S. A. Diddams, S. B. Papp, and K. J. Vahala, Nat. Photon. B 10, 316-320 (2016). 


\section{INFORMATIONAL FIFTH PAGE}

\section{REFERENCES}

1. P. Grelu, Nonlinear Optical Cavity Dynamics: From Microresonators to Fiber Lasers, (Wiley-VCH 2015).

2. M. Nakazawa, K. Suzuki, and H. A. Haus, "Modulational instability oscillation in nonlinear dispersive ring cavity", Phys. Rev. A. 38, 51935196 (1988).

3. S. Coen, and M. Haelterman, "Modulational Instability Induced by Cavity Boundary Conditions in a Normally Dispersive Optical Fiber", Phys. Rev. Lett. 79, 4139-4142 (1997)

4. M. Haelterman, S. Trillo, and S. Wabnitz, "Additive-modulationinstability ring laser in the normal dispersion regime of a fiber", Opt. Lett. 17, 745-747 (1992).

5. M. Tlidi, A. Mussot, E. Louvergneaux, G. Kozyreff, A. G. Vladimirov, and M. Taki, "Control and removal of modulational instabilities in lowdispersion photonic crystal fiber cavities", Opt. Lett. 32, 662 (2007).

6. S. B. Cavalcanti, J. C. Cressoni, H. R. da Cruz, and A. S. GouveiaNeto, "Modulation instability in the region of minimum group-velocity dispersion of single-mode optical fibers via an extended nonlinear Schrödinger equation", Phys. Rev. A 43, 6162-6165 (1991).

7. S. Pitois and G. Millot, "Experimental observation of a new modulational instability spectral window induced by fourth-order dispersion in a normally dispersive single-mode optical fiber", Opt. Commun. 226, 415-422 (2003).

8. J. D. Harvey, R. Leonhardt, S. Coen, and G. K. L. Wong, "Scalar modulation instability in the normal dispersion regime by use of a photonic crystal fiber", Opt. Lett. 28, 2225-2227 (2003).

9. F. Copie, M. Conforti, A. Kudlinski, S. Trillo, and A. Mussot, "Modulation instability in the weak dispersion regime of a dispersion modulated passive fiber-ring cavity", Opt. Express 25, 11283-11296 (2017).

10. A. Mussot, E. Louvergneaux, N. Akhmediev, F. Reynaud, L. Delage, and M. Taki, "Optical Fiber Systems Are Convectively Unstable", Phys. Rev. Lett. 101, 113904 (2008).

11. F. Leo, A. Mussot, P. Kockaert, P. Emplit, M. Haelterman, and M. Taki, "Nonlinear Symmetry Breaking Induced by Third-Order Dispersion in Optical Fiber Cavities", Phys. Rev. Lett. 110, 104103 (2013).

12. J. K. Jang, M. Erkintalo, S. G. Murdoch, and S. Coen, "Observation of dispersive wave emission by temporal cavity solitons", Opt. Lett. 39, 5503-5506 (2014).

13. P. Parra-Rivas, D. Gomila, F. Leo, S. Coen, and L. Gelens, "Third-order chromatic dispersion stabilizes Kerr frequency combs", Opt. Lett. 39, 2971-2974 (2014)

14. L. A. Lugiato and R. Lefever, "Spatial Dissipative Structures in Passive Optical Systems", Phys. Rev. Lett. 58, 2209-2211 (1987).

15. S. Coen, H. G. Randle, T. Sylvestre, and M. Erkintalo, "Modeling of octave-spanning Kerr frequency combs using a generalized mean-field Lugiato-Lefever model", Opt. Lett. 38, 37 (2013).

16. S. Coen, M. Haelterman, P. Emplit, L. Delage, L. M. Simohamed, and François Reynaud, "Bistable switching induced by modulational instability in a normally dispersive all-fibre ring cavity", J. Opt. B Quantum Semiclassical Opt. 1, 36 (1999).

17. F. Copie, M. Conforti, A. Kudlinski, and A. Mussot, "Competing Turing and Faraday Instabilities in Longitudinally Modulated Passive Resonators", Phys. Rev. Lett. 116, 143901 (2016).

18. M. Conforti, A. Mussot, A. Kudlinski, and S. Trillo, "Modulational instability in dispersion oscillating fiber ring cavities", Opt. Lett. 39, 4200 (2014).

19. S. Coen, M. Haelterman, P. Emplit, L. Delage, L. M. Simohamed, and F. Reynaud, "Experimental investigation of the dynamics of a stabilized nonlinear fiber ring resonator", J. Opt. Soc. Am. B 15, 2283 (1998).

20. Y. Okawachi, M. R. E. Lamont, K. Luke, D. O. Carvalho, M. Yu, M. Lipson, and A. L. Gaeta, "Bandwidth shaping of microresonator-based frequency combs via dispersion engineering", Opt. Lett. 39, 3535-3538 (2014).

21. C. Milián, A. V. Gorbach, M. Taki, A. V. Yulin, and D. V. Skryabin, "Solitons and frequency combs in silica microring resonators: Interplay of the Raman and higher-order dispersion effects", Phys. Rev. A 92,
033851 (2015).

22. K. Y. Yang, K. Beha, D. C. Cole, X. Yi, P. Del'Haye, H. Lee, J. Li, D. Y. Oh, S. A. Diddams, S. B. Papp, and K. J. Vahala, "Broadband dispersion-engineered microresonator on a chip", Nat. Photon. B 10, 316-320 (2016). 